\title{
NUEVA ESPECIE DE ROMANSCHULZIA (BRASSICACEAE) DEL ESTADO DE GUERRERO, MÉXICO
}

\author{
Rubí Bustamante y Rosa María Fonseca \\ Universidad Nacional Autónoma de México, Facultad de Ciencias, \\ Laboratorio de Plantas Vasculares, Apdo. postal 70-282, 04510 \\ México, D.F. México. tewamil@hotmail.com, rubunbura@yahoo.com.mx
}

\section{RESUMEN}

Se describe y se ilustra Romanschulzia guerrerensis, una nueva especie del estado de Guerrero. Se asemeja a $R$. apetala, $R$. subclavata y $R$. rzedowskii en que tienen flores apétalas, pero difiere de las dos primeras por presentar sépalos libres en lugar de connados y frutos sésiles en vez de estipitados. Se parece a $R$. rzedowskii en sus sépalos libres, anteras pequeñas y frutos sésiles y cilíndricos, pero difiere de ésta en tener frutos y pedúnculos reflejos vs. erectos y ascendentes. Se incluye una clave para las cuatro especies y una tabla en la cual se comparan las características de las mismas. $R$. guerrerensis crece en una zona templada y húmeda cerca del cerro Teotepec en Guerrero.

Palabras clave: Brassicaceae, Guerrero, México, Romanschulzia.

\begin{abstract}
Romanschulzia guerrerensis, a new species from the State of Guerrero (Mexico), is described and illustrated. The new species resembles $R$. apetala, $R$. subclavata and $R$. rzedowskii in having apetalous flowers, but differs from the first two by having free instead of connate sepals and sessile instead of stipitate fruits. It resembles $R$. rzedowskii in the having free sepals, small anthers, and sessile, terete fruits, but differs in the erect-ascending vs. reflexed fruits and fruiting pedicels. It grows in a temperate and humid zone near cerro Teotepec in Guerrero. A key to the four species and a table comparing their various morphological characters are provided.
\end{abstract}

Key words: Brassicaceae, Guerrero, Mexico, Romanschulzia. 


\section{INTRODUCCIÓN}

La familia Brassicaceae consta principalmente de hierbas y sufrútices, que en su mayoría son anuales; tal vez sea esa la razón por la cual han recibido poca atención por parte de los investigadores que hacen estudios florísticos. No obstante, se trata de un conjunto de aproximadamente 338 géneros y 3709 especies (Al-Shehbaz et al., 2006). Rzedowski y Rzedowski (2001) registraron 27 géneros para el Valle de México. En el estado de Guerrero, al momento se han encontrado Brassica rapa L., Cardamine bonariensis Pers., Capsella bursa-pastoris (L.) Medik., Halimolobos polysperma (E. Fourn.) O.E. Schulz, ahora conocida como Exhalimolobos polyspermus (E. Fourn.) Al-Shehbaz \& C.D. Bailey, de acuerdo con Bailey et al. (2007); Lexarzanthe mexicana (H.H. Iltis \& Al-Shehbaz) N. Diego \& Calderón, Raphanus sativus $\mathrm{L}$., Nasturtium officinale R. Br. (registrada por error, como Rorippa nasturtium-aquaticum) y R. pinnata (Sessé \& Moc.) Rollins (Fonseca, 2001; Diego-Pérez et al., 2001; Lozada-Pérez, 2003; Velázquez y Domínguez, 2003; Diego-Pérez y Calderón, 2004) y se espera localizar un mayor número de especies en particular en la zona templada del estado. La planta que ahora se da a conocer se encuentra en uno de los sitios de mayor altitud y más húmedos de la entidad.

Del estado de Guerrero se describió Romanschulzia mexicana Al-Shehbaz \& Iltis (1993); misma que para Diego-Pérez y Calderón (2004) pertenece a un género distinto, Lexarzanthe Diego-Pérez \& Calderón; sin embargo, Al-Shehbaz (comunicación personal) informa que, de acuerdo con estudios moleculares recientes, esta especie no difiere de Romanschulzia.

Romanschulzia guerrerensis Bustamante et Fonseca, sp. nov. Fig. 1.

Herbae erectae; caulis cylindricus et hispidus; folia sessilia, auriculata vel amplexicaulia, apice acuta, marginibus denticulatis vel subintegris; inflorescentiae racemosae terminales et axilllares; sepala 5 erecta, libera, petala absentia; fructus anguste cylindricus, leviter moniliformis, pedunculus erectus. Semina pallide fusca.

Hierbas de hasta $70 \mathrm{~cm}$ de longitud. Tallos verdes, cilíndricos, acanalados superficialmente, hasta $4 \mathrm{~mm}$ de diámetro, híspidos. Hojas simples, alternas, distribuidas a lo largo del tallo, sésiles; láminas verdes en la haz, verde grisáceas en el envés, oblongo lanceoladas a oblanceoladas, 4 a $10 \mathrm{~cm}$ de longitud, 2.2 a $4.5 \mathrm{~cm}$ de ancho, las hojas próximas a la inflorescencia de menor tamaño, ápice agudo a mucronado, margen denticulado a subentero, base amplexicaule y auriculada, textura 


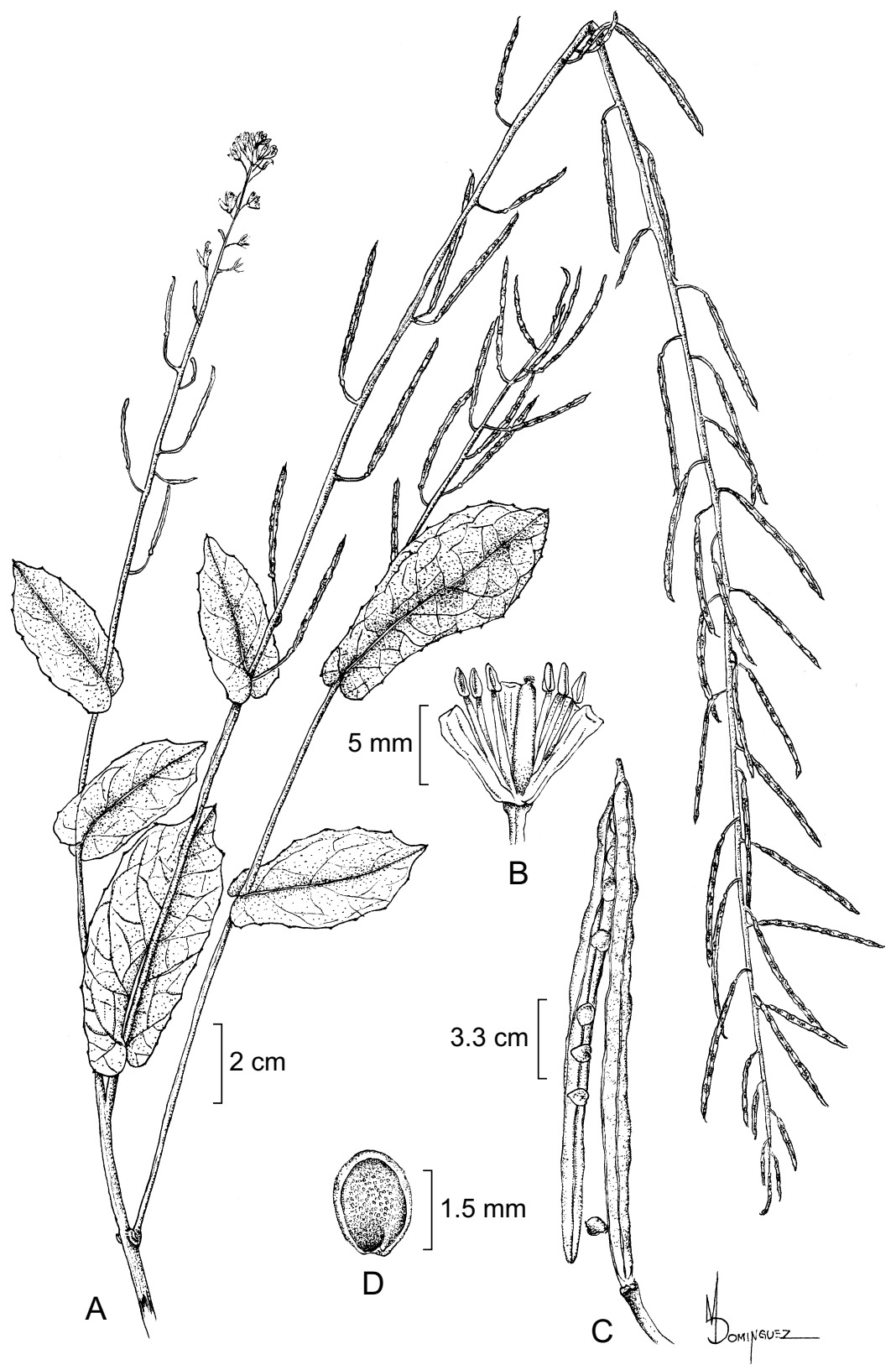

Fig. 1. Romanschulzia guerrerensis sp. nov. A. rama con hojas, flores y frutos; B. flor; C. fruto; D. semilla. 
membranácea, vena principal evidente, color crema, venación reticulada conspicua, ligeramente prominente en el envés, esparcidamente pubescentes, la haz menos que en el envés, principalmente sobre las venas, glabrescentes. Inflorescencias en racimos terminales sobre ramas laterales y eje principal, hasta de $35 \mathrm{~cm}$ de longitud; pedúnculos 5 a $11 \mathrm{~cm}$ de longitud; raquis cilíndrico. Flores con pedicelos de 2.7 a 4 mm de longitud; cáliz con 5 sépalos petaloides, libres, color crema, verdosos y con el ápice morado cuando jóvenes, venas conspicuas de color amarillo, espatulados, 2.8 $\mathrm{mm}$ de longitud, $1.2 \mathrm{~mm}$ de ancho, ápice truncado y emarginado o irregularmente dentado; pétalos ausentes; estambres 6, iguales, filamentos de $3.3 \mathrm{~mm}$ de longitud, anteras basifijas, con tecas divergentes, recurvadas al abrir, color verde, de 1 a 1.3 $\mathrm{mm}$ de longitud, $0.7 \mathrm{~mm}$ de ancho, nectarios presentes en la base de todos los estambres; ovario de color verde, cilíndrico, de $3.8 \mathrm{~mm}$ de longitud, $0.7 \mathrm{~mm}$ de ancho, glabro, bilocular, óvulos numerosos por cavidad, dirigidos hacia la base, 2 hileras en cada lóculo, estilo ausente, estigma discoide. Frutos tipo silicua, erectos, verdes, cilíndricos, ligeramente moniliformes, apiculados, de 30 a $35 \mathrm{~mm}$ de longitud, (1)1.5-1.6 mm de ancho, sésiles, glabros; pedúnculos ascendentes, de 5 a $8 \mathrm{~mm}$ de longitud. Semillas numerosas, biseriadas, color pardo claro con tintes rojizos, obovoides a elipsoides, de 1.3 a $1.5 \mathrm{~mm}$ de longitud, superficie menudamente ampollosa, glabras.

Tipo: México, Guerrero: municipio General Heliodoro Castillo, aproximadamente $2 \mathrm{~km}$ al este de Puerto del Gallo, por la carretera rumbo al cerro Teotepec, bosque de coníferas, alt. 2400 m, 12.VIII.2004, R. M. Fonseca y E. Velázquez 3193 (FCME), isotipos por distribuirse.

Los sépalos de esta especie tienen el aspecto petaloide ya señalado y se observó que al rehidratarlos adquieren la forma de un saco que se abre mediante una abertura longitudinal en la cara interna del sépalo. Rollins (1993) describe para Romanschulzia sépalos no sacciformes, a pesar de ello se decidió ubicar la presente especie dentro de este género, debido a que las otras características sí coinciden.

Las semillas aparentemente forman una hilera, que en realidad surgen de manera alterna de cada lado del septo, constituyendo dos series en cada lóculo.

A pesar de que la zona donde fue colectada esta planta ha sido ampliamente trabajada (Velázquez y Domínguez, 2003), sólo se cuenta con una colecta de cuatro ejemplares de la especie, debido probablemente a que se trata de una planta anual y se desarrolla en un sitio de difícil acceso. Es importante señalar que se desarrolla solamente en la orilla de un arroyo. 
Se colectó con flores y frutos en agosto, en bosque de coníferas con Abies religiosa y Pinus maximinoi, en una zona cercana al cerro Teotepec.

$R$. guerrerensis, al igual que algunos otros representantes del género, tiene flores apétalas pero se distingue por las silicuas erectas y sin ginóforo, pedúnculos ascendentes, tamaño de sépalos y de inflorescencia (ver Cuadro 1).

El taxon nuevo es similar a $R$. rzedowskii en aspectos como: sépalos libres, ausencia de ginóforo, silicua cilíndrica y anteras pequeñas; pero se distingue de ésta por las silicuas erectas, los pedicelos ascendentes de mayor longitud y el mayor

Cuadro 1. Diferencias morfológicas entre Romanschulzia guerrerensis y especies afines.

\begin{tabular}{|c|c|c|c|c|}
\hline & $\begin{array}{l}\text { R. apetala } \\
\text { Rollins }\end{array}$ & $\begin{array}{l}\text { R. subclavata } \\
\text { Rollins }\end{array}$ & $\begin{array}{l}\text { R. rzedowskii } \\
\text { Rollins }\end{array}$ & $\begin{array}{l}\text { R. guerrerensis } \\
\text { Bustamante et } \\
\text { Fonseca }\end{array}$ \\
\hline \multicolumn{5}{|l|}{ Flor } \\
\hline \multicolumn{5}{|l|}{ Sépalos } \\
\hline largo & (4) 6 a $8 \mathrm{~mm}$ & $3 \mathrm{a} 4 \mathrm{~mm}$ & $2.5 \mathrm{~mm}$ & $2.8 \mathrm{~mm}$ \\
\hline ancho & 2 a $3 \mathrm{~mm}$ & $1.5 \mathrm{a} 2 \mathrm{~mm}$ & $1.5 \mathrm{~mm}$ & $1.2 \mathrm{~mm}$ \\
\hline unión & $\begin{array}{l}\text { unidos en el } \\
\text { margen }\end{array}$ & $\begin{array}{l}\text { unidos en pares } \\
\text { en la base }\end{array}$ & libres & libres \\
\hline Anteras (largo) & ca. $2 \mathrm{~mm}$ & 1.8 a $2 \mathrm{~mm}$ & $>1 \mathrm{~mm}$ & $1 \mathrm{a} 1.3 \mathrm{~mm}$ \\
\hline $\begin{array}{l}\text { Inflorescencia } \\
\text { largo } \\
\text { pedicelos en flor }\end{array}$ & $\begin{array}{l}>70 \mathrm{~cm} \\
15 \mathrm{a} 25 \mathrm{~mm}\end{array}$ & $\begin{array}{l}100 \mathrm{~cm} \text { o más } \\
?\end{array}$ & $\begin{array}{l}\text { ca. } 10 \mathrm{~cm} \\
?\end{array}$ & $\begin{array}{l}\text { hasta } 35 \mathrm{~cm} \\
2.7 \text { a } 4 \mathrm{~mm}\end{array}$ \\
\hline \multicolumn{5}{|l|}{ Fruto (silicua) } \\
\hline ginóforo (largo) & 3 a $6 \mathrm{~mm}$ & 1.5 a $2 \mathrm{~mm}$ & ausente & ausente \\
\hline pedúnculos (largo) & $?$ & 5 a $15 \mathrm{~mm}$ & 4 a $6 \mathrm{~mm}$ & $5 \mathrm{a} 8 \mathrm{~mm}$ \\
\hline posición & $?$ & $\begin{array}{l}\text { erecta, pedicelos } \\
\text { extendidos o } \\
\text { ascendentes }\end{array}$ & $\begin{array}{l}\text { pendiente, } \\
\text { pedicelos } \\
\text { recurvados }\end{array}$ & $\begin{array}{l}\text { erecta, pedicelos } \\
\text { ascendentes }\end{array}$ \\
\hline forma & aplanada & $\begin{array}{l}\text { linear, a veces } \\
\text { subclavada, } \\
\text { cercanamente } \\
\text { cilíndrica }\end{array}$ & cilíndrica & cilíndrica \\
\hline largo & 20 a $30 \mathrm{~mm}$ & 15 a $25 \mathrm{~mm}$ & 25 a $30 \mathrm{~mm}$ & 30 a $35 \mathrm{~mm}$ \\
\hline diámetro & $2.5 \mathrm{a} 4 \mathrm{~mm}$ & $1.5 \mathrm{~mm}$ & $<2 \mathrm{~mm}$ & (1) $1.5 \mathrm{a} 1.6 \mathrm{~mm}$ \\
\hline
\end{tabular}


tamaño de la inflorescencia. La especie puede distinguirse de las afines mediante la siguiente clave:

1. Silicuas aplanadas de modo paralelo al septo, de más de $2 \mathrm{~mm}$ de diámetro R. apetala

1. Silicuas cilíndricas o subclavadas, no aplanadas, de menos de $2 \mathrm{~mm}$ de diámetro.

2. Silicuas pendientes; pedicelos recurvados; inflorescencias de aproximadamente $10 \mathrm{~cm}$ de largo R. rzedowskii

2. Silicuas erectas; pedicelos extendidos o ascendentes, inflorescencias de 30 cm o más de largo.

3. Silicuas de 15 a $25 \mathrm{~mm}$ de largo, con ginóforo de 1.5 a $2 \mathrm{~mm}$; sépalos de 3 a $4 \mathrm{~mm}$ de largo, unidos en pares en la base; anteras de 1.8 a $2 \mathrm{~mm}$ de largo R. subclavata

3. Silicuas de 30 a $35 \mathrm{~mm}$ de largo, sésiles; sépalos de $2.8 \mathrm{~mm}$ de largo, libres; anteras de 1 a $1.3 \mathrm{~mm}$ de largo R. guerrerensis

\section{AGRADECIMIENTOS}

Las autoras desean agradecer a la Maestra Graciela Calderón de Rzedowski quien tuvo la amabilidad de leer el manuscrito y darnos sus comentarios y observaciones; también a las Dras. Lourdes Rico y Nelly Diego la lectura crítica del documento y a Jorge Domínguez por la realización de la ilustración. Damos gracias de manera especial al Dr. Ihsan A. Al-Shehbaz por la revisión del manuscrito y por las valiosas observaciones que hizo para mejorar este trabajo.

\section{LITERATURA CITADA}

Al-Shehbaz, I. A. y H. H. Iltis. 1993. Romanschulzia mexicana (Brassicaceae), a remarkable new species from Guerrero, Mexico. Novon 3: 96-98.

Al-Shehbaz, I. A., M. A. Beilstein y E. A. Kellogg. 2006. Systematics and phylogeny of the Brassicaceae (Cruciferae): an overview. Plant Syst. Evol. 259: 89-120.

Bailey, C. D., I. A. Al-Shehbaz y G. Rajanikanth. 2007. Generic limits in tribe Halimolobeae and description of the new genus Exhalimolobos (Brassicaceae). Syst. Bot. 32(1): $140-156$. 
Diego-Pérez, N., S. Peralta-Gómez y B. Ludlow-Wiechers. 2001. El Jilguero. Bosque mesófilo de montaña. In: Diego-Pérez, N. y R. M. Fonseca (eds.). Estudios florísticos en Guerrero. No. 11. Facultad de Ciencias. Universidad Nacional Autónoma de México. México, D.F. 42 pp.

Diego-Pérez, N. y G. Calderón de Rzedowski. 2004. Un nuevo género de Cruciferae (Brassicaceae) del estado de Guerrero, México. Acta Bot. Mex. 68: 73-79.

Fonseca, R. M. 2001. Carrizal de Bravos. Bosque mesófilo de montaña. In: Diego-Pérez, N. y R. M. Fonseca (eds.). Estudios florísticos en Guerrero. No. 12. Facultad de Ciencias. Universidad Nacional Autónoma de México. México, D.F. 41 pp.

Lozada-Pérez, L., M. E. León, J. Rojas y R. de Santiago. 2003. Bosque mesófilo de montaña en El Molote. In: Diego-Pérez, N. y R. M. Fonseca (eds.). Estudios florísticos en Guerrero. No. 13. Facultad de Ciencias. Universidad Nacional Autónoma de México. México, D.F. 35 pp.

Velázquez, E. y E. Domínguez. 2003. Cerro Teotepec. In: Diego-Pérez, N. y R. M. Fonseca (eds.). Estudios florísticos en Guerrero. No. 15. Facultad de Ciencias. Universidad Nacional Autónoma de México. México, D.F. 37 pp.

Rollins, R. C. 1993. The Cruciferae of continental North America. Stanford University Press. Stanford, California. 976 pp.

Rzedowski, G. C. de, J. Rzedowski y colaboradores. 2001. Flora fanerogámica del Valle de México. 2a. ed., Instituto de Ecología, A. C. y Comisión Nacional para el Conocimiento y Uso de la Biodiversidad, Pátzcuaro (Michoacán). 1406 pp. 
